\title{
A role for cardiopulmonary exercise testing in detecting physiological changes underlying health status in Idiopathic pulmonary fibrosis: a feasibility study
}

\author{
R. Davis ${ }^{1}$, C. Dixon ${ }^{2}$, A. B. Millar ${ }^{1,2}$, N. A. Maskell ${ }^{2}$ and S. L. Barratt ${ }^{1,2^{*}}$
}

\begin{abstract}
Introduction: There is limited data available on the use of CPET as a predictive tool for disease outcomes in the setting of IPF. We investigated the feasibility of undertaking CPET and the relationship between CPET and quality of life measurements in a well-defined population of mild and moderate IPF patients.
\end{abstract}

Methods: A prospective, single-centre observational study.

Results: Thirty-two IPF patients (mild $n=23$, moderate $n=9$ ) participated in the study, $n=13$ mild patients attended for repeat CPET testing at 12 months. At baseline, total K-BILD scores and total IPF-PROM scores significantly correlated with $6 \mathrm{MWT}$ distance, but not with baseline FVC \% predicted, TLco \% predicted, baseline or minimum $\mathrm{SpO}_{2}$. $\mathrm{VO}_{2}$ peak/kg at AT positively correlated with total scores, breathlessness/activity and chest domains of the K-BILD questionnaire $(p<0.05) . \mathrm{VO}_{2}$ peak significantly correlated with total IPF PROM scores and wellbeing domains $(p<0.05)$, with a trend towards statistical significance for total IPF-PROM and $\mathrm{VO}_{2}$ peak/kg at anaerobic threshold $(p=0.06)$.

There was a statistically significant reduction in FVC\% predicted at 12 months follow up, although the mean absolute decline was $<10 \%(p<0.05)$. During this period $\mathrm{VO}_{2}$ peak significantly reduced $(21.6 \mathrm{ml} / \mathrm{kg} / \mathrm{min} \pm 2.9 \mathrm{vs} 19.1 \pm 2.8$; $p=0.017$ ), with corresponding reductions in total K-BILD and breathlessness/activity domains that exceeded the MCID for responsiveness. Lower baseline $\mathrm{VO}_{2}$ peak $/ \mathrm{kg}$ at anaerobic threshold correlated with greater declines in total K-BILD scores $(r=-0.62,0.024)$ at 12 months. Whilst baseline FVC\% predicted or TLCo \% predicted did not predict change in health status,

Conclusion: We have shown that it is feasible to undertake CPET in patients with mild to moderate IPF. CPET measures of $\mathrm{VO}_{2}$ peak correlated with both baseline and change in K-BILD measurements at 1 year, despite relatively stable standard lung function (declines of $<10 \%$ in FVC), suggesting its potential sensitivity to detect physiological changes underlying health status.

Keywords: Cardiopulmonary exercise testing, Idiopathic pulmonary fibrosis, Health status

*Correspondence: Shaney.Barratt@nbt.nhs.uk

${ }^{1}$ Academic Respiratory Unit, School of Clinical Sciences, Southmead

Hospital, University of Bristol, Learning and Research Building, Bristol BS10

$5 \mathrm{NB}$, UK

Full list of author information is available at the end of the article

\section{Introduction}

Idiopathic Pulmonary Fibrosis (IPF) is a progressive fibrosing lung disease of increasing prevalence [1], associated with median survival of only $3-5$ years from diagnosis $[2,3]$. Disease heterogeneity continues to present challenges for clinicians with regards to prognostication 
and optimal timings for lung transplantation and/or advanced care planning $[4,5]$. In the setting of large-scale clinical trials, a decline in forced vital capacity (FVC) has been used as a primary outcome measure $[6,7]$ and as a surrogate for mortality, although this has not been universally endorsed $[8,9]$.

Cardiopulmonary exercise testing (CPET) is the considered the gold standard for evaluating maximal/ symptom-limited exercise tolerance, encompassing respiratory, cardiovascular and musculoskeletal assessments, in a controlled laboratory environment [10-12].

However there is limited data available on the use of CPET as a predictive tool for disease outcomes in the setting of IPF. A recent systematic review identified only two small-scale prospective studies that investigated the role of CPET in the prognostication in IPF $[13,14]$ and concluded that there was insufficient evidence to support its use in facilitating 'real world' clinical decisions at the current time.

We have investigated the feasibility of undertaking CPET in a population of mild and moderate IPF patients in terms of the attrition of participants, information on safety data, and willingness to engage with the study protocol. Secondary end-points included: the change in CPET parameters over a 1 year period and the correlation between baseline CPET parameters and change in lung function, 6MWT and health status at 1 year.

We hypothesised that CPET would be feasible in population of mild to moderate IPF patients and more sensitive to change in patient's health status than $6 \mathrm{MWT}$, FVC or TLco.

\section{Methods}

\section{Study design}

This was a prospective, single-centre observational study undertaken at a large secondary care institution in the UK, providing secondary and tertiary care to patients with Interstitial Lung disease (ILD) within the SouthWest of England. The study was approved by the Health Research Authority and Research Ethics Committees (IRAS 223450).

\section{Study subjects}

Patients with a multidisciplinary team (MDT) diagnosis of IPF, based upon the American Thoracic Society/European Respiratory Society 2018 guidelines [15], were prospectively recruited to the study between June 2018 and May 2019. Written informed consent was obtained from each patient.

Patients were divided into a 'mild' or 'moderate' category dependent on their baseline Forced Vital Capacity (FVC $\geq 50 \%-<80 \%$ : moderate; FVC $\geq 80 \%$ mild). Those patients in the 'mild' disease group would undertake both a baseline and repeat CPET at 12 months. It was decided by the study committee, due to the uncertainty of the ability of those with reduced lung function to perform a maximal exercise test, that those in the 'moderate' disease group would undertake only a baseline CPET test.

\section{Inclusion and exclusion criteria}

Inclusion criteria were an MDT consensus diagnosis of IPF, male or female aged $\geq 40$ years, TLCO $\geq 50 \%$ predicted and $\mathrm{FVC} \geq 50 \%$ with written informed consent for study participation.

Key exclusion criteria were: FEV1/FVC ratio $<$ the lower limit of normal, mobility issues preventing the participant to undertake cycle ergometry, history of myocardial infarction (MI) within 6 months or unstable angina within 1 month, uncontrolled arrhythmias causing symptoms or haemodynamic compromise, history of recent syncope (within last 6 months), acute thrombosis within previous 6 months, cognitive impairment/ inability to perform CPET, severe or untreated arterial hypertension (>200 $\mathrm{mmHg}$ systolic at rest, $>120 \mathrm{mmHg}$ diastolic) and patients using oxygen treatment.

\section{Participant testing Pulmonary function testing}

Pulmonary function tests were performed in accordance with ATS/ERS guidelines [16], using the European Community of Coal and Steel (ECCS) reference equations [17]. Forced expiratory volume during first second of expiration $\left(\mathrm{FEV}_{1}\right)$, forced vital capacity (FVC), and transfer factor for carbon monoxide (TLCO) were undertaken at baseline (within 4 weeks of CPET) and at 12 months ( \pm 4 weeks) (nSpire HDpft, nSpire Health $\mathrm{GmbH}$, Germany). The MRC score, age (years), height (meters), and body weight of the patients (kilograms) were also recorded.

\section{6 minute walking test (6MWT)}

A 6MWT was performed at baseline (and within 3 months of CPET) according to ATS guidelines [18], using the Enright reference equation [19]. The following data were collected and analysed: distance achieved (metres), oxygen saturation at the initiation of the test, the minimum saturation level, percentage of theoretical distance achieved and at the end of the test.

\section{Cardiopulmonary exercise testing (CPET)}

CPET was performed using a standardized protocol in accordance with the American Thoracic Society/ American College of Chest Physicians (ATS/ACCP) statement [20], using Wasserman [21] and Jones [22] reference equations. All patients underwent a symptom-limited CPET to exhaustion or intolerability with 
an electromagnetically braked cycle ergometer (Ergoselect 100, ergoline $\mathrm{GmbH}$, Germany) using a ramp protocol over 8-12 $\mathrm{min}$. The protocol included $3 \mathrm{~min}$ of rest, 2 min of unloaded cycling (at 60 revolutions per $\mathrm{min}$ ), followed by a progressively increasing work rate in a ramp fashion, and a recovery period (patient dependent). The work rate increment for each ramped exercise test was selected depending on the patient's level of daily activity (either 5 or $10 \mathrm{~W} / \mathrm{min} \mathrm{ramp}$ ).

Subjects were asked to maintain a rate of 60 revolutions per minute throughout the exercise period. Several markers were used to determine if a maximal effort test was performed; a respiratory exchange ratio (RER; $\left.\mathrm{VCO}_{2} / \mathrm{VO}_{2}\right) \geq 1.1$, maximum heart rate ( $\mathrm{HR}$ max $)>90 \%$ of maximum predicted HR (220-age), maximum minute ventilation during exercise $>85 \%$ predicted based on MVV at rest (maximum voluntary ventilation) and a plateau in $\mathrm{VO}_{2}$ with an increased workload. CPET could be discontinued at the discretion of the supervising attendant if clinically indicated.

Cardiopulmonary data were collected and analysed with nSpire Zan 600 USB system (nSpire Health GmbH, Germany).

The following parameters were recorded:

- Peak oxygen consumption $\left(\mathrm{VO}_{2}\right.$ peak, $\left.\mathrm{ml} / \mathrm{kg} / \mathrm{min}\right)$,

- Oxygen consumption at anaerobic threshold

- Carbon dioxide production $\left(\mathrm{VCO}_{2}\right)$

- Peak minute ventilation (VE peak)(marker of ventilatory function during exercise),

- $\mathrm{VE} / \mathrm{VCO}_{2}$ slope as derived from the above values (reflects changes in ventilatory drive)

- Peripheral capillary oxygen saturation $\mathrm{SpO}_{2}$ (marker of hypoxaemia indicating possible ventilatory limitation to exercise)

- Peak power output (W)(marker of musculoskeletal function)

- Heart rate (HR) (marker of cardiac function during exercise),

- Breathing reserve (BR)

Anaerobic threshold was determined noninvasively through the plot of $\mathrm{VCO}_{2}$ versus $\mathrm{VO}_{2}$ (V-slope method). Predicted minute ventilation was automatically calculated by the software as a function of maximal voluntary ventilation (MVV), where $\mathrm{MVV}=\mathrm{FEV} 1$ $\mathrm{L} \times 40$. The BR was automatically calculated from the software as the difference between the maximum voluntary ventilation at rest and the peak ventilation. The $\%$ predicted $\mathrm{VO}_{2}$ peak and \% theoretical $\mathrm{VO}_{2}$ peak at AT were not determined in this study as it was felt that populations deriving existing reference equations and normal standards were not representative of the male predominant and elderly population, characteristic of IPF patients/populations.

\section{Health status questionnaires and patient-reported outcomes}

Patients were asked to complete the King's Brief ILD questionnaire (K-BILD) [23] and IPF-Patient reported outcome measure (IPF-PROM) [24], in addition to the Leicester Cough visual analogue scale (VAS) for cough [25] and Bristol VAS for breathlessness and fatigue [26], at baseline and at 12 months.

The K-BILD is a self-completed health status questionnaire that comprises 15 items in three domains of psychological, breathlessness and activities and chest symptoms. The K-BILD scoring system implements logit transformation of raw item response scores to provide total score ranges of $0-100$, where 100 represents best health status. The minimally important clinical difference (MCID) for the logit version of the K-BILD questionnaire is 5 for total K-BILD, 6 for Psychological, 7 for Breathlessness and activities and 11 for Chest symptoms [27].

The IPF-PROM [24] is a self-completed 12 item health status questionnaire that measures the physical and psychological experience of breathlessness; emotional wellbeing and fatigue. The questionnaire has been validated in terms of face and content validity. The scores range from 12 to 48, where 48 indicates worst health status.

\section{Outcomes}

We wished to study the feasibility of undertaking CPET in a population of mild and moderate IPF patients: the attrition of participants, information on safety data, and willingness to engage with the study protocol. Secondary end-points included: the change in CPET parameters over a 1 year period and the correlation between baseline CPET parameters and change in lung function, $6 \mathrm{MWT}$ and health status at 1 year.

\section{Statistical analysis}

Categorical variables are reported as absolute numbers and percentages. Normality of continuous data was initially verified using D'Agostino and Pearson normality test. Mean and standard deviations (SD) were used to describe parametric data; median and interquartile range (IQR, in brackets) for non-parametric data. Differences among two groups were verified by $\mathrm{t}$-test with Welch's correction for continuous parametric data or Mann-Whitney U for non-parametric data. Fisher's exact test was used to compare two categorical variables (with small sample size), whilst $x^{2}$-test was used to compare multiple categorical variables or larger sample sizes. Paired t-tests were used for comparison of parametric variables from baseline to 12 months or Wilcoxon 
in the case of non-parametric data. Pearson's correlation was used to determine correlations between parametric variables. Data were analysed using GraphPad Prism version 8.0. A $p$ value of $<0.05$ was considered statistically significant.

\section{Results}

\section{Study population}

Forty-two consecutive IPF patients were prospectively enrolled to the study. Four patients subsequently withdrew consent, 1 patient died and 5 patients developed exclusion criteria prior to commencement of the study. A further 5 patients did not complete the study and were lost to follow up (4 mild, 1 moderate). The final population studied thus consisted of 27 patients (mild $n=19$ and moderate $\mathrm{n}=8$ ) (Fig. 1). Patients were predominantly male $(\mathrm{n}=22,82 \%)$ with a mean age of 75 years $( \pm 6.0$ years $)$ and were symptomatic at baseline with a median MRC breathlessness score of 2 (IQR 2-3). Approximately one third $(33 \%, n=9)$ of patients (mild $\mathrm{n}=5$, moderate $\mathrm{n}=4$ ) received antifibrotics during the observational period. At completion of 1 year follow up, all patients remained alive.

\section{Feasibility}

There was excellent willingness to engage with the study protocol.

All patients achieved a RER $>1.1$ and the vast majority of patients also achieved $>80 \%$ of their maximal predicted heart rate $(25 / 27,93 \%)$ and /or had limited breathing reserve, providing corroboration that patients performed at maximal effort.

At baseline, all participants achieved the anaerobic threshold during testing and at 1 year follow up only one patient failed to achieve the anaerobic threshold.

Breathlessness and fatigue were the most commonly cited reasons for terminating CPET. Of patients completing the study ( $\mathrm{n}=27 ; 19$ mild and 9 moderate IPF), baseline CPET was terminated due to breathlessness in $37 \%$ (10/27), the majority of which had mild IPF $(90 \%, 9 / 10)$. Leg/muscle fatigue was cited as a reason for terminating CPET in $63 \%$ patients (17/27), of which 59\% (10/17) had mild disease. There were no significant differences in the reasons for terminating CPET between those that completed and did not complete follow-up.

At 1 year repeat CPET, 54\% (7/13) described breathlessness as the reason for stopping and 38\% (5/13) muscle

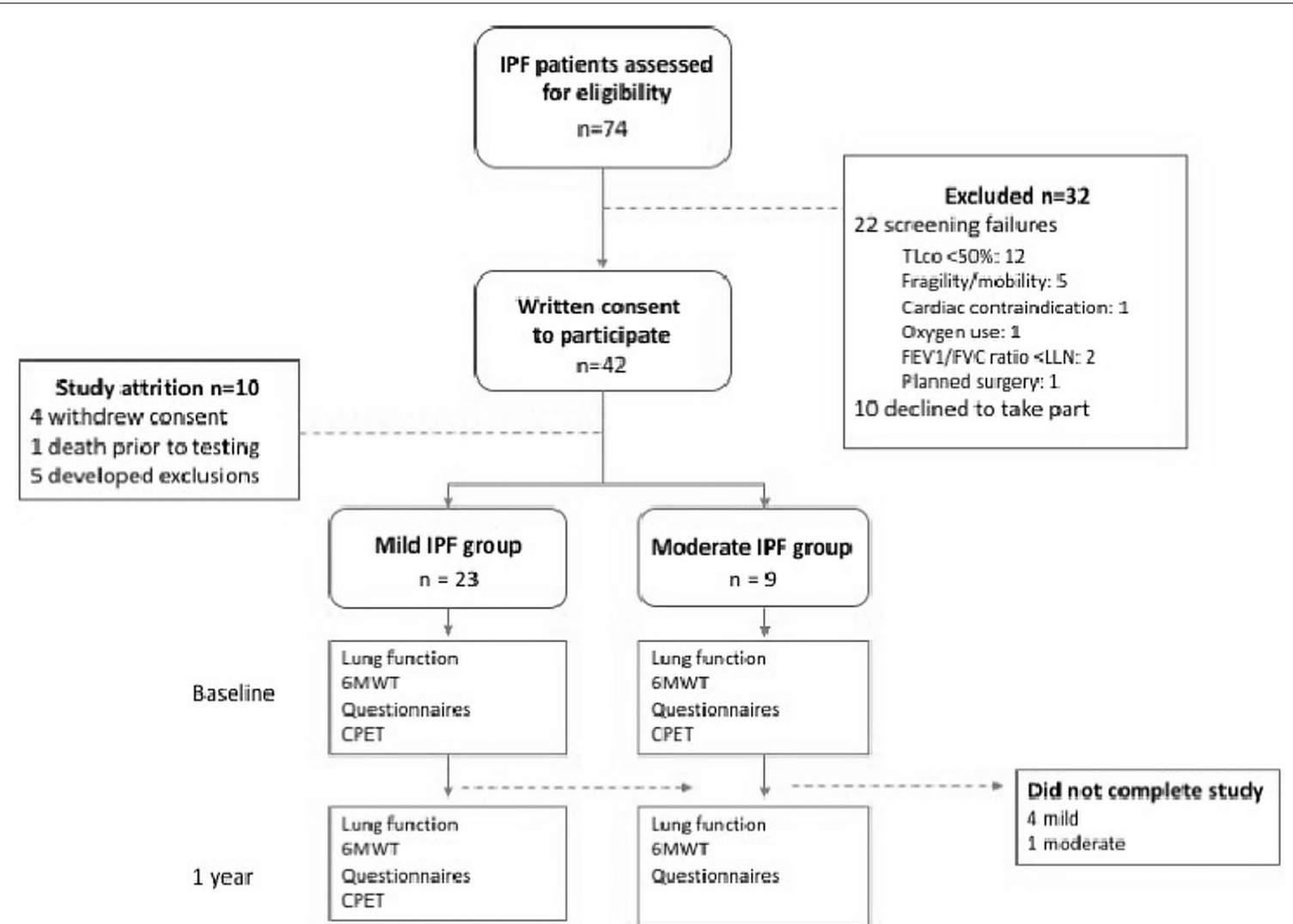

Fig. 1 Consort diagram of study participants. TLCo transfer factor; FEV1/FVC $<$ LLN, Forced expiratory volume in $1 \mathrm{~s} /$ Forced vital capacity $<$ lower limit of normal; IPF idiopathic pulmonary fibrosis; 6MWT six minute walk test; CPET cardiopulmonary exercise testing 
fatigue. A dry mouth was cited as the main contributing reason for stopping in one patient.

One patient described dizziness related to his breathlessness during CPET but no other adverse events were recorded. There were no serious adverse events.

\section{Baseline measurements between mild and moderate IPF groups}

Baseline demographics between mild and moderate IPF groups were statistically comparable (Table 1). As per a priori subgroup definitions, participants in the moderate IPF group had a statistically lower baseline FVC $\%$ predicted compared to those in the mild IPF group (mild $99 \%$ predicted \pm 10.0 , range $85-125 \%$ predicted) vs moderate $70 \%$ predicted \pm 5.1 , range $62-75 \%$ predicted, $p<0.0001)$. In keeping with these findings there was a trend towards a lower TLco in those within the moderate IPF group (mild $63 \%$ predicted \pm 9.5 ), range $50-83 \%$ predicted vs moderate $57 \%$ predicted \pm 6.2 , range $50-65 \%$ predicted, $p=0.055)$. No significant difference in the $6 \mathrm{MWT}$ distance measured between mild and moderate groups was observed.

Patients with moderate disease had numerically lower total K-BILD, chest symptom and psychological domain scores compared to those with mild disease, although values were not statistically different (total K-BILD mild disease $67( \pm 10.3)$ vs moderate disease $60( \pm 6.6)$, $p=0.058)$. There were no significant differences in VAS scores of cough, breathlessness or fatigue between mild and moderate IPF groups or IPF-PROM measurements.

Whilst baseline CPET values were all within 'normal' published ranges [20, 28], peak minute ventilation (\% predicted) was significantly higher for those with moderate IPF compared to those with mild disease (mild 71.0\% $( \pm 13.9)$ vs moderate $82.9 \%( \pm 12.4, p=0.045)$ (Table 1$)$.

Of the baseline CPET parameters measured, $\mathrm{VO}_{2}$ peak/ $\mathrm{kg}$ at anaerobic threshold positively correlated with total scores $(\mathrm{r}=0.42, p=0.03)$, breathlessness/activity $(\mathrm{r}=0.47, p=0.014)$ and chest domains $(\mathrm{r}=0.44, p=0.02)$ of the K-BILD questionnaire (Pearson's correlation). Similarly, total IPF PROM scores and wellbeing domains significantly correlated with $\mathrm{VO}_{2}$ peak $(\mathrm{r}=-0.43, p=0.02$ and $\mathrm{r}=-0.44, p=0.02$ ), with a trend towards statistical significance for total IPF-PROM and $\mathrm{VO}_{2}$ peak $/ \mathrm{kg}$ at anaerobic threshold $(p=0.06) . \mathrm{VE} / \mathrm{VCO}_{2}$ at anaerobic threshold also correlated with total K-BILD score $(\mathrm{r}=0.39 ; p=0.001)$ at baseline, although there were no significant correlations with the individual domains of the questionnaire or IPF-PROM.

Total K-BILD scores $(\mathrm{r}=0.44, p=0.03)$ and total IPFPROM scores $(r=-0.43, p=0.03)$ both significantly correlated with $6 \mathrm{MWT}$ distance, but not with baseline FVC $\%$ predicted (Total K-BILD, $p=0.14$; Total IPF-PROM $p=0.50$ ), TLco $\%$ predicted (Total K-BILD $p=0.16$; Total IPF-PROM $p=0.32$ ), baseline or minimum $\mathrm{SpO}_{2}$ (Total K-BILD $p=0.25$ and $p=0.32$, respectively, Total IPF PROM $p=0.53$ and $p=0.55$, respectively). There were no significant correlations between baseline CPET parameters and VAS scores $(p>0.05)$.

\section{Measurements at 1 year follow up Total IPF cohort}

At one year of follow up, the mean reduction in FVC and TLco \% predicted for the whole IPF cohort $(\mathrm{n}=27)$ was $-3.6 \%( \pm 7.1, p=0.02)$ and $-3.2 \%( \pm 7.5, p=0.04)$ respectively (Additional File 1: Table 1). Whilst statistically significant, values were below those deemed clinically significant $[29,30]$. There was no significant reduction in $6 \mathrm{MWT}$ distance achieved (mean reduction $4.8 \mathrm{~m} \pm 34.7, p=0.50$; mean reduction in $6 \mathrm{MWT}$ distance as $\%$ theoretical distance $0.2 \%( \pm 7.9, p=0.92)$. There was a statistically significant reduction in the breathlessness and activity domain scores of the K-BILD questionnaire from baseline to 1 year, suggesting worse health status $(-4.8 \pm 10.2, p=0.02)$, although this did not reach the published MCID for responsiveness for this domain [27]. There were no statistically significant differences in the VAS scores for cough, breathlessness and fatigue or in the IPF-PROM from baseline to one year $(p>0.05)$.

\section{Follow up of mild IPF group with repeat CPET}

Thirteen patients from the mild IPF group returned for repeat CPET at 1 year; the coronavirus COVID-19 pandemic prohibited the return of the remaining six patients at the one year follow-up time point as planned. All but one patient achieved anaerobic threshold.

Upon repeat CPET testing there were statistically significant declines in the $\mathrm{VO}_{2}$ peak $(21.6 \mathrm{ml} / \mathrm{kg} / \mathrm{min} \pm 2.9$ vs $19.1 \pm 2.8 ; p=0.017), \mathrm{VO}_{2}$ peak at $\mathrm{AT}(14.2 \mathrm{ml} /$ $\mathrm{kg} / \mathrm{min} \pm 3.2$ vs $11.8 \pm 1.6, p=0.044) \mathrm{VE}$ peak $(75.3$ $\mathrm{L} / \mathrm{min} \pm 20.9$ vs $66.1 \pm 21.6 ; p=0.007)$, peak work (106.9 W \pm 26.3 vs $90.8 \pm 25.9 ; p=0.022)$ heart rate response $(142.3 \mathrm{bpm} \pm 24.0$ vs $133 \pm 22.3 ; p=0.040)$ and increased breathing reserve (BRmax) $(21.8 \mathrm{~L} / \mathrm{min}(12.4-$ $34.2)$ vs $33.8(20.2-55.7) ; p=0.0002)$, compared to baseline values (Table 2).

There was a statistically significant reduction in FVC\% predicted at 12 months, although the mean absolute decline was $<10 \%$ (baseline FVC $98.8 \%$ predicted \pm 8.5 vs follow up FVC 93.4\% predicted \pm 10.3 , $p=0.01)$. In these same patients, statistically significant reductions in breathlessness/activity $(-7.2 \pm-10.8$; $p=0.033)$ and chest $(-9.6 \pm-15.0 ; p=0.040)$ domain scores of the K-BILD questionnaire were observed, with a trend towards statistical significance for reduction in 
Table 1 Baseline characteristics of IPF participants

\begin{tabular}{|c|c|c|c|c|}
\hline Characteristic & Overall $n=27$ & Mild $n=19$ & Moderate $n=8$ & $p$ value \\
\hline Age (years) (mean, SD) & $75( \pm 6.0)$ & $75( \pm 6.6)$ & $74( \pm 4.3)$ & 0.440 \\
\hline Gender (male $n, \%$ ) & $22,82 \%$ & $14,74 \%$ & $8,100 \%$ & 0.280 \\
\hline \multicolumn{5}{|l|}{ Smoking history $(\mathrm{n}, \%)$} \\
\hline Current & $0,0 \%$ & $0,0 \%$ & $0,0 \%$ & 0.552 \\
\hline Never & $10,37 \%$ & $9,47 \%$ & $1,12.5 \%$ & \\
\hline Ex-smoker & $17,63 \%$ & $10,53 \%$ & $7,87.5 \%$ & \\
\hline BMI (kg/m²) (mean, SD) & $28.5( \pm 4.5)$ & $28.9( \pm 5.0)$ & $27.7( \pm 2.9)$ & 0.585 \\
\hline MRC (n) & & & & 0.964 \\
\hline $0-1$ & 4 & 3 & 1 & \\
\hline 2 & 16 & 11 & 5 & \\
\hline 3 & 7 & 5 & 2 & \\
\hline $4-5$ & 0 & 0 & 0 & \\
\hline Antifibrotics $(n, \%)$ & $9,33 \%$ & $5,26 \%$ & $4,50 \%$ & 0.375 \\
\hline Co-morbidities & & & & $n / s$ \\
\hline Gastro-oesophageal reflux & 11 & 6 & 5 & \\
\hline Hypertension & 10 & 7 & 3 & \\
\hline Coronary artery disease & 10 & 4 & 6 & \\
\hline Diabetes & 3 & 1 & 2 & \\
\hline \multicolumn{5}{|l|}{ Lung function parameters (mean, SD) } \\
\hline $\mathrm{FVC}(\mathrm{L})$ & $2.96( \pm 0.72)$ & $3.11( \pm 0.78)$ & $2.61( \pm 0.46)$ & 0.050 \\
\hline FVC (\% predicted) & $91( \pm 16.0)$ & $99( \pm 10.0)$ & $70( \pm 5.1)$ & $<0.0001$ \\
\hline FEV1/FVC ratio & $78( \pm 7.9)$ & $76( \pm 7.8)$ & $82( \pm 7.2)$ & 0.080 \\
\hline TLco \% predicted & $61( \pm 9.0)$ & $63( \pm 9.5)$ & $57( \pm 6.2)$ & 0.055 \\
\hline 6MWT (mean, SD) & $n=25$ & $\mathrm{n}=18$ & $\mathrm{n}=7$ & \\
\hline Distance achieved (m) & $350( \pm 63.3)$ & $349( \pm 66.6)$ & $354( \pm 58.6)$ & 0.827 \\
\hline \% theoretical distance $(\mathrm{m})$ & $78( \pm 16.3)$ & $78( \pm 16.5)$ & $76( \pm 17.0)$ & 0.821 \\
\hline CPET (mean, SD) & $n=27$ & $n=19$ & $\mathrm{n}=8$ & \\
\hline $\mathrm{VO}_{2}$ peak $/ \mathrm{kg}(\mathrm{ml} / \mathrm{kg} / \mathrm{min})^{*}$ & $20.9( \pm 3.9)$ & $20.6( \pm 4.2)$ & $21.7( \pm 3.1)$ & 0.489 \\
\hline $\mathrm{VO}_{2} / \mathrm{kg}$ at $\mathrm{AT}(\mathrm{ml} / \mathrm{kg} / \mathrm{min})$ & $13.6( \pm 3.5)$ & $13.8( \pm 3.6)$ & $13.0( \pm 3.2)$ & 0.585 \\
\hline VE peak (L/min) & $69.9( \pm 21.1)$ & $69.0( \pm 21.7)$ & $72.1( \pm 20.9)$ & 0.731 \\
\hline VE peak (\% predicted) & $74.5( \pm 14.4)$ & $71.0( \pm 13.9)$ & $82.9( \pm 12.4)$ & 0.045 \\
\hline VENCOO 2 at AT & $28.2( \pm 3.1)$ & $28.7( \pm 3.3)$ & $27.2( \pm 2.4)$ & 0.201 \\
\hline Minimum $\mathrm{O}_{2}$ saturation during CPET(\%) & $91( \pm 4.7)$ & $91( \pm 5.4)$ & $92( \pm 2.8)$ & 0.723 \\
\hline Peak work rate $(\mathrm{W})$ & $104.8( \pm 26.4)$ & $103.6( \pm 29.4)$ & $107.6( \pm 18.7)$ & 0.673 \\
\hline Peak work (\% predicted) & $44( \pm 8.6)$ & $44( \pm 9.5)$ & $43( \pm 6.1)$ & 0.565 \\
\hline $\mathrm{HR}(\mathrm{bpm})$ & $141( \pm 21.8)$ & $141( \pm 21.2)$ & $139( \pm 24.5)$ & 0.793 \\
\hline HR (\% predicted) & $97( \pm 15.1)$ & $98( \pm 15.0)$ & $95( \pm 16.2)$ & 0.711 \\
\hline BR max (L/min); median (IQR) & $26.5(18.4-32.5)$ & $28.8(18.8-33.3)$ & $20.4(12-24.9), n=7$ & 0.083 \\
\hline K-BILD questionnaire (mean, SD) & $n=27$ & $n=19$ & $n=8$ & \\
\hline Total & $65( \pm 9.7)$ & $67( \pm 10.3)$ & $60( \pm 6.6)$ & 0.058 \\
\hline Psychological domain & $69( \pm 17.8)$ & $72( \pm 17.8)$ & $61( \pm 15.7)$ & 0.128 \\
\hline Breathlessness and activity domain & $56( \pm 12.6)$ & $57( \pm 13.6)$ & $52( \pm 9.4)$ & 0.267 \\
\hline Chest symptoms domain & $78( \pm 17.7)$ & $81( \pm 18.2)$ & $71( \pm 14.6)$ & 0.133 \\
\hline IPF-PROM questionnaire (mean, SD) & $n=27$ & $\mathrm{n}=19$ & $n=8$ & \\
\hline Total & $20( \pm 4.3)$ & $20( \pm 4.6)$ & $21( \pm 3.5)$ & 0.337 \\
\hline Physical breathlessness & $5( \pm 1.5)$ & $5( \pm 1.4)$ & $5( \pm 1.5)$ & 0.264 \\
\hline Psychological breathlessness & $5( \pm 1.3)$ & $5( \pm 1.2)$ & $6( \pm 1.5)$ & 0.121 \\
\hline Well-being & $5( \pm 2.0)$ & $5( \pm 2.2)$ & $5( \pm 1.3)$ & 0.662 \\
\hline Energy & $5( \pm 1.5)$ & $5( \pm 1.7)$ & $5( \pm 0.8)$ & 0.565 \\
\hline
\end{tabular}


Table 1 (continued)

\begin{tabular}{lllll}
\hline Characteristic & Overall $\mathbf{n}=\mathbf{2 7}$ & Mild $\mathbf{n}=\mathbf{1 9}$ & Moderate $\mathbf{n}=\mathbf{8}$ & $\boldsymbol{p}$ value \\
\hline VAS Cough (cm) (median, (IQR)) & $1.7(0.8-2.8)$ & $1.5(0.2-2.6)$ & $2.3(1.4-3.0)$ & 0.135 \\
Bristol VAS breathlessness (cm) & $1.9(0.8-3.3)$ & $1.8(0.8-3.4)$ & $2.4(1.1-4.7)$ & 0.630 \\
Bristol VAS fatigue (cm) & $3.7(1.1-5.1)$ & $3.7(1.1-5.1)$ & $3.8(1.4-6.3)$ & 0.457 \\
\hline All
\end{tabular}

All data shown as mean with standard deviation(SD) unless otherwise stated. All visual analogue scores presented as median with interquartile range (IQR). ${ }^{*}$ Referenced $\mathrm{VO}_{2}$ peak $/ \mathrm{kg}$ for a 75 year old man would be $26.5 \mathrm{ml} / \mathrm{kg} / \mathrm{min}$ (based on the linear regression equation male $=-0.42 \times$ age $(y e a r s)+58(\mathrm{Schneider}$, Lung 2013 [28]). years years; SD standard deviation; $n$ number; $\%$ percentage; FVC forced vital capacity; $L$ litres; FEV1 forced expiratory volume in one second, TLco transfer factor; $m$ metres, $H R$ heart rate; $M R C$ medical research council dyspnoea score; $V A S$ visual analogue score; $c m$ centimetres; $b p m$ beats per minute; $W$ Work; $O_{2}$ oxygen; $L$ litres; 6MWT 6 min walk test; BMI body mass index; CPET cardiopulmonary exercise test; $K$-BILD King's Brief interstitial lung disease questionnaire; IPF-PROM idiopathic pulmonary fibrosis patient reported outcome measure. Unpaired t-test with Welch's correction was used to compare parametric data of mild and moderate groups, whilst Mann Whitney u was used for non-parametric data. Fisher's exact test was used to compare categorical data. A $p<0.05$ was considered statistically significant

Table 2 Baseline and 1 year follow up data for patients within mild group (those with matched tests)

\begin{tabular}{|c|c|c|c|}
\hline CPET parameters & Baseline $(n=13)$ & Follow up $(n=13)$ & $p$ value \\
\hline $\mathrm{VO}_{2}$ peak $(\mathrm{ml} / \mathrm{kg} / \mathrm{min})$ & $21.6 \pm 2.9$ & $19.1 \pm 2.8$ & 0.017 \\
\hline $\mathrm{VO}_{2}$ peak at $\mathrm{AT}(\mathrm{ml} / \mathrm{kg} / \mathrm{min})$ & $14.2 \pm 3.2$ & $11.8 \pm 1.6, n=12$ & 0.044 \\
\hline VE peak (L/min) & $75.3 \pm 20.9$ & $66.1 \pm 21.6$ & 0.007 \\
\hline VE peak \% pred & $75.5 \pm 13.2$ & $65.9 \pm 12.2$ & 0.007 \\
\hline VEN $\mathrm{NCO}_{2}$ at AT & $29.7 \pm 3.1$ & $31 \pm 4.6, n=12$ & 0.353 \\
\hline Minimum $\mathrm{O}_{2}$ saturation during CPET (\%) & $91.5 \pm 5.5$ & $87.9 \pm 6.6, n=12$ & 0.182 \\
\hline Peak Work (W) & $106.9 \pm 26.3$ & $90.8 \pm 25.9$ & 0.022 \\
\hline Peak Work (\% predicted) & $44.3 \pm 6.9$ & $37.7 \pm 8.5$ & 0.002 \\
\hline $\mathrm{HR}(\mathrm{bpm})$ & $142.3 \pm 24.0$ & $133 \pm 22.3$ & 0.040 \\
\hline HR (\% predicted) & $98.7 \pm 16.9$ & $91.8 \pm 16.8$ & 0.022 \\
\hline$B R \max (\mathrm{L} / \min )($ median, $(\mathrm{IQR}))$ & $21.8(12.4-34.2)$ & $33.8(20.2-55.7)$ & 0.0002 \\
\hline \multicolumn{4}{|l|}{ 6MWT } \\
\hline Distance achieved (m) & $346.9 \pm 73.8$ & $340.8 \pm 72.4$ & 0.563 \\
\hline \% theoretical distance $(\mathrm{m})$ & $76.4 \pm 18.3$ & $76.0 \pm 16.8$ & 0.872 \\
\hline \multicolumn{4}{|l|}{ Lung function } \\
\hline FVC \% predicted & $98.8 \pm 8.5$ & $93.4 \pm 10.3$ & 0.010 \\
\hline TLco \% predicted & $62.3 \pm 9.4$ & $59.3 \pm 11.8$ & 0.161 \\
\hline
\end{tabular}

$\mathrm{VO}_{2}$ peak peak oxygen consumption/kg; $A T$ anaerobic threshold; $V E$ minute ventilation; $V \mathrm{CO}_{2}$ carbon dioxide output; $V C \mathrm{O}_{2}$ ventilatory equivalent for carbon dioxide; $V E / V \mathrm{CO}_{2}$ BR max breathing reserve; $W$ work; bpm beats per minute; \% percentage; IQR interquartile range; $n$ number; $6 M W T 6$ min walk test; $F V C$ Forced Vital Capacity; TLco transfer factor; $m$ metres; \% percentage; CPET cardiopulmonary exercise testing. All values are shown as mean \pm standard deviation, unless otherwise stated. Paired t-test was used for parametric data, whilst Wilcoxon matched pairs signed rank test was used for non-parametric data. A $p<0.05$ was considered statistically significant

the total B-ILD score $(-5.6 \pm-10.4 ; p=0.077)$ at follow up. Notably, the mean unit change of total K-BILD and breathlessness/activity domain scores exceeded the minimally clinically important difference previously reported (5 and 7 unit change respectively) [27], with $5 / 13$ patients achieving the MCID for total K-BILD score and 8/13 for the breathlessness/activity domains (Table 3).

There were no statistically significant differences in the VAS scores for cough, breathlessness or fatigue VAS score from baseline to one year $(p>0.05)$. There was statistically significant worsening in the psychological experience of breathlessness as reported by the IPFPROM $(0.8 \pm 1.2, p=0.044)$, although the clinical significance of this small statistical change is not clear.

Reductions in K-BILD scores observed at 12 months were correlated with baseline CPET measurements. Lower baseline $\mathrm{VO}_{2}$ peak/ $\mathrm{kg}$ at anaerobic threshold correlated with greater declines in total K-BILD scores $(r=-0.62,0.024)$ and psychological domains of the K-BILD at follow up $(\mathrm{r}=-0.63, p=0.022)$. No other baseline CPET parameters significantly correlated with change in K-BILD score in this small cohort, including peak work rate. Furthermore, there was no significant correlation with the baseline FVC\% predicted $(p=0.70)$ 
Table 3 Change in K-BILD, IPF-PROM and Visual analogue scores of mild IPF patients with repeat CPET at 1 year follow up

\begin{tabular}{lll}
\hline & $\begin{array}{l}\text { Unit change } \\
\text { (mean, SD) } \mathbf{n}=\mathbf{1 3}\end{array}$ & $\boldsymbol{p}$ value \\
\hline K-BILD questionnaire & & \\
Total & $-5.6( \pm 10.4)$ & 0.077 \\
Psychological domain & $-5.2( \pm 18.8)$ & 0.304 \\
Breathlessness and activity domain & $-7.2( \pm 10.8)$ & 0.033 \\
Chest symptoms domain & $-9.6( \pm 15.0)$ & 0.040 \\
IPF-PROM & & \\
Total & $1.5( \pm 0.9)$ & 0.109 \\
Physical breathlessness & $0.5( \pm 1.4)$ & 0.189 \\
Psychological breathlessness & $0.8( \pm 1.2)$ & 0.044 \\
Well-being & $-0.2( \pm 1.2)$ & 0.656 \\
Energy & $0.4( \pm 1.4)$ & 0.337 \\
VAS Cough (cm) median & -1.6 & 0.391 \\
Bristol VAS breathlessness (cm) median & 0.0 & 0.716 \\
Bristol VAS fatigue (cm) median & -0.1 & 0.956 \\
\hline
\end{tabular}

Results shown as mean change in questionnaire score with standard deviations (SD), unless otherwise stated. Paired t-test used for parametric data and Wilcoxon matched pairs signed rank test for non-parametric data. K-BILD King's Brief interstitial lung disease questionnaire; IPF-PROM Idiopathic pulmonary fibrosis-patient reported outcome measure; VAS visual analogue scale; $\mathrm{cm}$ centimetres. $p$ value $<0.05$ considered statistically significant

or TLco\% predicted $(p=0.62)$ and change in K-BILD score (Pearson's correlation).

\section{Discussion}

CPET is considered the gold standard for evaluating exertional dyspnoea and exercise intolerance in patients with cardiorespiratory conditions [31], yet currently lacks a defined role in the management of ILD [31, 32]. A recent systematic review by our group highlighted the insufficient available evidence to support the use of CPET in disease prognostication in ILD, emphasising that heterogeneity in terms of the ILD populations studied and the retrospective nature of the majority of published studies limited the conclusions that could be drawn [33]. Furthermore, the minimally clinically important differences for CPET parameters in ILD have not been established.

Our study has shown that CPET can be undertaken in both mild and moderate populations of IPF patients, without any significant adverse events, although study attrition was high and complicated by COVID-19 restrictions, such that only $64 \%$ patients completing the protocol.

Our prospective data suggests that baseline CPET $\mathrm{VO}_{2}$ peak is associated with clinically-meaningful patientperceived reduction in health status at 1 year, in spite of comparatively stable lung function parameters $(<10 \%$ decline in FVC and $<15 \%$ decline in TLco). $\mathrm{VO}_{2}$ peak is an integrated measure of respiratory, cardiovascular and neuromuscular function [20]. In a progressive disease such as IPF, the finding of reduced exercise performance at one year was not a surprising one. However, results suggest that this reduction was not as a consequence of ventilatory limitation. There was no change in the CPET ventilatory mode and the development of cardiac $+/$ - pulmonary vascular dysfunction was not apparent. One possible explanation might be that patients became more deconditioned with reduced activity levels in response to their perceived worsening of breathlessness. El Nagger et al. [34] have previously shown that $\mathrm{VO}_{2}$ peak correlated with health status of IPF patients at baseline as determined by the St Georges questionnaire but longitudinal changes in CPET parameters and associated health status were not explored. In our cohort, $\mathrm{VO}_{2}$ peak during exercise correlated with patient reported outcome measures at baseline, it significantly declined at 12 months and also correlated with the change in patient reported health status at 12 months.

Existing literature conflicts as to whether $\mathrm{VO}_{2}$ peak might predict disease outcomes in IPF; peak $\mathrm{VO}_{2}$ thresholds ranging from $<8.3$ to $<14.2 \mathrm{ml} / \mathrm{kg} / \mathrm{min}[13,14,35]$ have been reported to predict mortality in IPF, whilst others studies have failed to identify any significant association [36-38]. Ongoing follow up of our prospective cohort will be used to further study the use of baseline CPET parameters to predict longer-term outcomes in these patients.

It is recognised that this study has limitations. Firstly, and perhaps most importantly, the study was conducted on a relatively small and homogenous sample of patients. This limits the overall generalisability of results, particularly in terms of feasibility of CPET across IPF phenotypes; for example those with exercise induced pulmonary hypertension versus those with relatively normal pulmonary vascular response to exercise, and the risk of Type II error may be relatively high.

The vast majority of patients had mild IPF (72\%) with a median MRC score of 2; again limiting the generalisability of results. Whilst it would have been preferable to have a broader range of symptomatic patients, exercise in patients with high MRC scores would be very restricted leading to early completion of tests before the limit of pulmonary and cardiovascular systems had been reached [39] and thus negatively influencing the results. A further limitation of the study was that almost a quarter of patients enrolled in the study developed exclusions to CPET or were lost to follow up; a factor that will be helpful to inform power calculations for future studies involving CPET as an outcome measure. It was decided by the study committee in the planning of the protocol that due to the uncertainty of the ability and the safety of those with reduced lung 
function to perform a maximal exercise test, those in the 'moderate' disease group would undertake only a baseline CPET test. In retrospect, it would have been more valuable to undertake repeat CPET on all enrolled participants. With the experience gained from this study, this is something that could be explored in the future.

It is recognised by the authors that the selection of reference value equations can have a significant impact on the interpretation of CPET results [20], for example, the 220-age equation may underestimate HRmax in older adults [20]. The authors have used reference equations to contextualise the results where appropriate, recognising that many existing reference equations are not fully representative of the male dominant and elderly population characteristic of IPF populations.

Finally, the COVID-19 pandemic adversely affected the ability to perform follow-up CPET testing, particularly in this highly vulnerable group of individuals; consequently the resulting sample size was small.

\section{Conclusion}

In conclusion, our study provides the initial data to support the feasibility of CPET in at least mild-moderate populations of IPF and the ability of repeated CPET to assess the change in health status over time. This may be clinically applied in the future to assess the response to pharmacological or non-pharmacological interventions from the patient's perspective. Future work should concentrate on examining the relationship between CPET parameters, lung function and CTderived measures of disease, establishing the MCID for longitudinal change in CPET in ILD.

\section{Supplementary Information}

The online version contains supplementary material available at https://doi. org/10.1186/s12890-021-01520-8.

Additional file 1: Table 1. Change in Lung function, K-BILD, IPF-PROM and Visual analogue scores of all IPF patients at 1 year follow up. Paired t-test or Wilcoxon paired signed rank test.

\section{Acknowledgements}

We would like to acknowledge Jason Viner, Head of the lung physiology department at North Bristol Trust for all his support throughout this project.

\section{Authors' contributions}

The study was conceptualised by ABM and SLB. RD undertook the study under the supervision of SLB, ABM and NAM. CD performed the CPET tests. SLB wrote the first draft of the manuscript and performed the analysis. All authors ( $R D, C D, A B M, N M, S L B)$ contributed to the final draft of the manuscript. All authors read and approved the final manuscript.

\section{Funding}

No funding was required for this study.

\section{Availability of data and materials}

The datasets used and/or analysed during the current study available from the corresponding author on reasonable request.

\section{Declarations}

Consent to publish

Not applicable.

\section{Informed consent}

Informed written consent was obtained for all participants in the study. The study was approved by the Health Research Authority and Research Ethics Committees (IRAS 223450). All methods were carried out in accordance with relevant guidelines and regulations.

\section{Competing interests}

Richard Davis(RD) is an employee of Boehringer Ingelheim (BI). This project was undertaken independently by RD as part of a MsC with the University of Bristol. BI had no part in the concept or undertaking of this project. Dr Barratt has received consultancy fees from Boehringer Ingeleheim, separate to the submitted work. No other conflicts of interests are declared.

\section{Author details}

${ }_{1}^{1}$ Academic Respiratory Unit, School of Clinical Sciences, Southmead Hospital, University of Bristol, Learning and Research Building, Bristol BS10 5NB, UK.

${ }^{2}$ Bristol Interstitial Lung Disease Service, North Bristol NHS Trust, Bristol, UK.

Received: 23 December 2020 Accepted: 12 April 2021

Published online: 05 May 2021

\section{References}

1. Navaratnam V, Fleming KM, West J, Smith CJ, Jenkins RG, Fogarty A, et al. The rising incidence of idiopathic pulmonary fibrosis in the U.K. Thorax. 2011;66(6):462-7.

2. Raghu G, Weycker D, Edelsberg J, Bradford WZ, Oster G. Incidence and prevalence of idiopathic pulmonary fibrosis. Am J Respir Crit Care Med. 2006;174(7):810-6.

3. Ley B, Collard HR, King TE. Clinical course and prediction of survival in idiopathic pulmonary fibrosis. Am J Respir Crit Care Med. 2011;183(4):431-40.

4. Mura M, Porretta MA, Bargagli E, Sergiacomi G, Zompatori M, Sverzellati $\mathrm{N}$, et al. Predicting survival in newly diagnosed idiopathic pulmonary fibrosis: a 3-year prospective study. Eur Respir J. 2012;40(1):101-9.

5. Schroedl CJ, Yount SE, Szmuilowicz E, Hutchison PJ, Rosenberg SR, Kalhan R. A qualitative study of unmet healthcare needs in chronic obstructive pulmonary disease. A potential role for specialist palliative care? Ann Am Thorac Soc. 2014;11(9):1433-8.

6. Richeldi L, du Bois RM, Raghu G, Azuma A, Brown KK, Costabel U, et al. Efficacy and safety of nintedanib in idiopathic pulmonary fibrosis. $N$ Engl J Med. 2014;370(22):2071-82.

7. King TE, Bradford WZ, Castro-Bernardini S, Fagan EA, Glaspole I, Glassberg MK, et al. A phase 3 trial of pirfenidone in patients with idiopathic pulmonary fibrosis. N Engl J Med. 2014;370(22):2083-92.

8. Wells AU, Behr J, Costabel U, Cottin V, Poletti V, Richeldi L, et al. Hot of the breath: mortality as a primary end-point in IPF treatment trials: the best is the enemy of the good. Thorax. 2012;67(11):938-40.

9. King TE, Safrin S, Starko KM, Brown KK, Noble PW, Raghu G, et al. Analyses of efficacy end points in a controlled trial of interferon-gamma $1 \mathrm{~b}$ for idiopathic pulmonary fibrosis. Chest. 2005;127(1):171-7.

10. Layton AM, Armstrong HF, Kim HP, Meza KS, D’Ovidio F, Arcasoy SM. Cardiopulmonary exercise factors predict survival in patients with advanced interstitial lung disease referred for lung transplantation. Respir Med. 2017;126:59-67.

11. Sue DY, Wasserman K. Impact of integrative cardiopulmonary exercise testing on clinical decision making. Chest. 1991;99(4):981-92.

12. Palange P, Ward SA, Carlsen KH, Casaburi R, Gallagher CG, Gosselink R, et al. Recommendations on the use of exercise testing in clinical practice. Eur Respir J. 2007;29(1):185-209. 
13. Triantafillidou C, Manali E, Lyberopoulos P, Kolilekas L, Kagouridis K, Gyftopoulos $\mathrm{S}$, et al. The role of cardiopulmonary exercise test in IPF prognosis. Pulm Med. 2013;2013:514817.

14. Vainshelboim B, Oliveira J, Fox BD, Kramer MR. The prognostic role of ventilatory inefficiency and exercise capacity in idiopathic pulmonary fibrosis. Respir Care. 2016;61(8):1100-9.

15. Raghu G, Remy-Jardin M, Myers JL, Richeldi L, Ryerson CJ, Lederer DJ, et al. Diagnosis of idiopathic pulmonary fibrosis. An official ATS/ERS/JRS/ALAT clinical practice guideline. Am J Respir Crit Care Med. 2018;198(5):e44-68.

16 Graham BL, Steenbruggen I, Miller MR, Barjaktarevic IZ, Cooper BG, Hall GL, et al. Standardization of Spirometry 2019 Update. An Official American Thoracic Society and European Respiratory Society Technical Statement. Am J Respir Crit Care Med. 2019;200(8):e70-88.

17 Quanjer PH, Tammeling GJ, Cotes JE, Fabbri LM, Matthys H, Pedersen OF, et al. Symbols, abbreviations and units. Working Party Standardization of Lung Function Tests, European Community for Steel and Coal. Eur Respir J Suppl. 1993;16:85-100.

18. Laboratories ACoPSfCPF. ATS statement: guidelines for the six-minute walk test. Am J Respir Crit Care Med. 2002;166(1):111-7.

19. Enright $P L$, Sherrill DL. Reference equations for the six-minute walk in healthy adults. Am J Respir Crit Care Med. 1998;158(5 Pt 1):1384-7.

20. Society AT, Physicians ACoC. ATS/ACCP Statement on cardiopulmonary exercise testing. Am J Respir Crit Care Med. 2003;167(2):211-77.

21. Wasserman K, Stringer WW, Casaburi R, Koike A, Cooper CB. Determination of the anaerobic threshold by gas exchange: biochemical considerations, methodology and physiological effects. Z Kardiol. 1994;83(Suppl 3):1-12.

22. Jones NL, Makrides L, Hitchcock C, Chypchar T, McCartney N. Normal standards for an incremental progressive cycle ergometer test. Am Rev Respir Dis. 1985;131(5):700-8.

23. Patel AS, Siegert RJ, Creamer D, Larkin G, Maher TM, Renzoni EA, et al. The development and validation of the King's Sarcoidosis Questionnaire for the assessment of health status. Thorax. 2013;68(1):57-65.

24. Russell A, Wickremasinghe $M$, Renzoni E, Adamali H, Boreal Z FS, Maher TM, Ng Man Kwong G, Saketkoo LA, Fleming S, Cullinan P. The idiopathic pulmonary fibrosis patients reported outcome measure (IPF-PROM) is reliable and valid for use in populations with IPF. British Thoracic Society: Thorax; 2018. p. A47.

25. Key AL, Holt K, Hamilton A, Smith JA, Earis JE. Objective cough frequency in idiopathic pulmonary fibrosis. Cough. 2010;6:4

26. Yates H, Adamali HI, Maskell N, Barratt S, Sharp C. Visual analogue scales for interstitial lung disease: a prospective validation study. QJM. 2018.

27. Sinha A, Patel AS, Siegert RJ, Bajwah S, Maher TM, Renzoni EA, et al. The King's Brief Interstitial Lung Disease (KBILD) questionnaire: an updated minimal clinically important difference. BMJ Open Respir Res. 2019;6(1):e000363.
28. Schneider J. Age dependency of oxygen uptake and related parameters in exercise testing: an expert opinion on reference values suitable for adults. Lung. 2013;191(5):449-58.

29. Collard HR, King TE, Bartelson BB, Vourlekis JS, Schwarz MI, Brown KK. Changes in clinical and physiologic variables predict survival in idiopathic pulmonary fibrosis. Am J Respir Crit Care Med. 2003;168(5):538-42.

30. Flaherty KR, Andrei AC, Murray S, Fraley C, Colby TV, Travis WD, et al. Idiopathic pulmonary fibrosis: prognostic value of changes in physiology and six-minute-walk test. Am J Respir Crit Care Med. 2006;174(7):803-9.

31. Molgat-Seon Y, Schaeffer MR, Ryerson CJ, Guenette JA. Cardiopulmonary exercise testing in patients with interstitial lung disease. Front Physiol. 2020;11:832.

32. Raghu G, Collard HR, Egan JJ, Martinez FJ, Behr J, Brown KK, et al. An official ATS/ERS/JRS/ALAT statement: idiopathic pulmonary fibrosis: evidence-based guidelines for diagnosis and management. Am J Respir Crit Care Med. 2011;183(6):788-824

33. Barratt SL, Davis R, Sharp C, Pauling JD. The prognostic value of cardiopulmonary exercise testing in interstitial lung disease: a systematic review. ERJ Open Res. 2020;6(3).

34. El Naggar T, Said AM, Sharkawy SH, RH R. Evaluation of the questionnaires validity in assessing the severity of idiopathic pulmonary fibrosis in correlation with high-resolution computed tomography, lung diffusion, and cardiopulmonary exercise tests. Egypt J Bronchol 2017;11:141-8.

35. Fell CD, Liu LX, Motika C, Kazerooni EA, Gross BH, Travis WD, et al. The prognostic value of cardiopulmonary exercise testing in idiopathic pulmonary fibrosis. Am J Respir Crit Care Med. 2009;179(5):402-7.

36. Wallaert B, Guetta A, Wemeau-Stervinou L, Tercé G, Valette M, Nevière R, et al. Prognostic value of clinical exercise testing in idiopathic pulmonary fibrosis. Rev Mal Respir. 2011;28(3):290-6.

37. Miki K, Maekura R, Hiraga T, Okuda Y, Okamoto T, Hirotani A, et al. Impairments and prognostic factors for survival in patients with idiopathic pulmonary fibrosis. Respir Med. 2003;97(5):482-90.

38. van der Plas MN, van Kan C, Blumenthal J, Jansen HM, Wells AU, Bresser P. Pulmonary vascular limitation to exercise and survival in idiopathic pulmonary fibrosis. Respirology. 2014;19(2):269-75.

39. O'Donnell DE, Ora J, Webb KA, Laveneziana P, Jensen D. Mechanisms of activity-related dyspnea in pulmonary diseases. Respir Physiol Neurobiol. 2009;167(1):116-32.

\section{Publisher's Note}

Springer Nature remains neutral with regard to jurisdictional claims in published maps and institutional affiliations.
Ready to submit your research? Choose BMC and benefit from:

- fast, convenient online submission

- thorough peer review by experienced researchers in your field

- rapid publication on acceptance

- support for research data, including large and complex data types

- gold Open Access which fosters wider collaboration and increased citations

- maximum visibility for your research: over 100M website views per year

At BMC, research is always in progress.

Learn more biomedcentral.com/submissions 